\title{
Association between the Average Ratio of Platelet Volume and the Presence of Mural Thrombus in Post-Myocardial Infarction
}

\author{
Fernando Augusto Alves da Costa ${ }^{1,2}$ \\ Editorial referring to the article: Association between Mean Platelet Volume-to-Lymphocyte Ratio and the Presence of Apical Mural Thrombus in \\ Post-Myocardial Infarction Patients \\ Clínica Paulista de Doenças Cardiovasculares, ${ }^{1}$ São Paulo, SP - Brazil. \\ Hospital Beneficência Portuguesa de São Paulo, ${ }^{2}$ São Paulo, SP-Brazil.
}

Cardiovascular disease is a serious publichealth problem, responsible for $31 \%$ of all deaths worldwide. Population growth, urbanization and the lack of proper policies to control modifiable risk factors lead to an increased number in cases. ${ }^{1}$ Associated with this, we have new factors such as stress, pollution, income, unemployment, among others, which, associated with classical factors, increase the speed of atherosclerosis development. Acute ischemic syndromes are some of the worst consequences of the evolution of atherosclerosis, with mortality rates varying according to the type of infarction, place of care and the healthcare resources available.

Among the types of infarction, those with ST segment elevation (STEMI), particularly in the anterior wall, present peculiarities in their natural history, and may lead to complications, such as sudden death, heart failure and embolic events, usually due to the formation of apical thrombus (AT) in the left ventricle.

The pathophysiology of AT formation has been studied for decades and we do not have laboratory elements to determine the patients that may have this condition. Complications due to the presence of AT are serious, with systemic embolization to different territories and the possibility of complications, death and permanent sequelae. ${ }^{2}$

Detection of AT formation after STEMI is essential and its presence indicates treatments that aim to reduce the probability of embolization. Many of these thrombi

\section{Keywords}

Cardiovascular Diseases/prevention and control; Public Health Program;Risk Factors; Stress; Atherosclerosis; Mortality; ST Elevation Myocardial Infarction; Thrombosis. develop at different times and, therefore, are not diagnosed, greatly increasing the risk of complications for these patients.

The study of platelet aggregation and coagulation cascade, which are more active during and after acute coronary syndrome, points to this possibility, but we do not have any elements that may signal this condition. ${ }^{3}$

Literature data show that increased platelet volume (MPV) makes them more active from a metabolic and enzymatic point of view and the platelet-to-lymphocyte ratio (PLR) is known as an inflammatory marker that has been widely studied in other diseases, such as cancer, chronic kidney disease and coronary artery disease.

In a very elegant way, a study was designed using these two variables, called MPVLR, and their association with AT formation. The findings showed that MPVLR was significantly increased in patients with thrombus in the left ventricle after myocardial infarction, compared with patients with no thrombus, with $82.1 \%$ sensitivity and $70.2 \%$ specificity in the ROC analysis, representing a predictor of AT formation. ${ }^{4}$

This is the first study that determined the use of MPVLR as a predictor of thrombus formation in the left ventricle after acute myocardial infarction in the anterior wall, through an easy-to-apply methodology and the findings seem to be a predictor of the possibility of AT development. ${ }^{4}$

There are study limitations due to the small number of study participants, because it is retrospective, conducted in a single center and with limitations where the authors recognize that the platelet counting methodology should be improved, in addition to further MPVLR testing at other times during the infarction, mainly to assess the 
maintenance of results and interference of drugs used in these patients, such as antiplatelet agents, statins, and others that may alter the MPV.

A great step has been taken towards a large multicenter study that may confirm these initial data and thereby determine the possibility of developing AT after STEMI in the anterior wall, and it may be studied in other cardiac diseases with potential for embolization.

The detection of this possibility and early and effective treatment may change the natural history of the disease, protecting patients from serious complications, reducing mortality due to the evolution of these diseases.

\section{References}

1. Organização Mundial de Saúde. Doença Cardiovascular. [Acessado em 30/08/2020] Disponível em: https://www.who.int/news-room/fact-sheets/ detail/cardiovascular-diseases-(cvds) acessado em 30/08/2020

2. McCarthy CP, Vaduganathan M, McCarthy KJ, Januzzi JL Jr, Bhatt DL, McEvoy JW. Left Ventricular Thrombus After Acute Myocardial Infarction: Screening, Prevention, and Treatment. JAMA Cardiol. 2018;3(7):642-9. doi:10.1001/jamacardio.2018.1086

3. Langford EJ, Wainwright RJ, Martin JF. Platelet activation in acute myocardial infarction and unstable angina is inhibited by
We have seen a steady growth in cardiovascular mortality over the past few decades. Technological and drug-related advances, capacity-building at the services and adherence to treatment have greatly helped reducing mortality.

Prevention of cardiovascular diseases on all stages of human life through the control of risk factors still needs greater adherence and better results, negatively impacting the reduction of development and complications, such as acute ischemic syndromes. ${ }^{1}$

The use of MPVLR may be useful at first to prevent embolic complications in acute coronary syndromes.

nitric oxide donors. Arterioscler Thromb Vasc Biol. 1996;16(1):51-5. doi:10.1161/01.atv.16.1.51

4. Koseoglu C, Kurmus O. Association between Mean Platelet Volume-to-Lymphocyte Ratio and the Presence of Apical Mural Thrombus in Post-Myocardial Infarction Patients. Int. J. Cardiovasc. Sci. [Internet]. [cited 2020 Sep 09]. Available from: http://www.scielo.br/scielo.php?script=sci_arttext\&pid=S2359. 56472020005007202\&lng=en. In press 2020. Epub Aug 07, 2020. https://doi.org/10.36660/ijcs.20190140. 